\title{
Experimental investigation of the effect of spatial aggregation on reproductive success in a rewardless orchid
}

\author{
Antonina I. Internicola $\cdot$ Nicolas Juillet • \\ Ann Smithson · Luc D. B. Gigord
}

Received: 4 November 2005/ Accepted: 2 August 2006/ Published online: 29 August 2006

(C) Springer-Verlag 2006

\begin{abstract}
Plant reproductive success within a patch may depend on plant aggregation through pollinator attraction. For rewardless plants that lack rewards for pollinators, reproductive success may rely strongly on the learning abilities of pollinators. These abilities depend on relative co-flowering rewarding and rewardless plant species spatial distributions. We investigated the effect of aggregation on the reproductive success of a rewardless orchid by setting up 16 arrays in a factorial design with two levels of intraspecific aggregation for both a rewardless orchid and a rewarding co-flowering species. Our results show that increasing aggregation of both species negatively influenced the reproductive success of the rewardless plants. To our knowledge, this is the first experimental study demonstrating negative effects of aggregation on reproductive success of a rewardless species due both to its own spatial aggregation and that of a co-flowering rewarding species. We argue that pollinator learning behaviour is the key driver behind this result.
\end{abstract}

Communicated by Jacqui Shykoff.

The authors declare that the experiments presented here comply with the current laws applicable in the country in which they were performed (France).

A. I. Internicola $\cdot$ N. Juillet $\cdot$ L. D. B. Gigord $(\bowtie)$ Department of Ecology and Evolution, Biology Building,

University of Lausanne, 1015 Lausanne, Switzerland

e-mail: Luc.Gigord@unil.ch

A. Smithson

Department of Biological Sciences, Hatherly Laboratories,

University of Exeter, Exeter, Devonshire, UK
Keywords Rewarding species ·

Dactylorhiza sambucina - Muscari neglectum .

Plant-pollinator interactions $\cdot$ Learning behaviour

\section{Introduction}

In plant communities both positive and negative interactions between plants, through competition and facilitation, can take place simultaneously (Holzapfel and Mahall 1999). These interactions can affect plant reproduction (Moeller 2004), survival (Tirado and Pugnaire 2003), growth (Holzapfel and Mahall 1999), and, therefore, the dynamics of plant populations (Tirado and Pugnaire 2003). Competitive or facilitative effects can occur both intra- and inter-specifically (Rathcke 1983), and processes underlying these interactions can be related to resource use or to pollinator visitation frequency (Rathcke 1983; Kennedy and Gray 1993). Facilitation among plants can operate by a diversity of mechanisms (Callaway 1995), but particularly in terms of reproductive success through mutual attraction of pollinators (Thomson 1978, 1981, 1982; Waser and Real 1979). In such circumstances interactions between plants of a given species could be influenced by the spatial aggregation of individuals of this species and also by those of co-flowering species. Contrary to competition for resources, where interactions are mainly with spatially close neighbours, animal-mediated interactions among plants can act at a much larger scale (Thomson 1983; Moeller 2004), up to the population, community, or even landscape scale. For instance, pollinators prefer dense flowering stands (Kunin 1993; Kennedy and Gray 1993) and may visit dense patches more often than those where plants are 
more dispersed, increasing plant reproductive success. Therefore, spatial patterns of plants and species distribution within communities are likely to be of fundamental importance to animal-mediated plant reproductive success (Pacala 1997).

Rewardlessness is generally rare in Angiosperms but widespread within the Orchidaceae, where about onethird of the species lack reward (van der Pijl and Dodson 1966; Ackerman 1986). The large majority of rewardless orchids are nectarless food frauds (Ackerman 1986). Their pollen is not available as a reward because it is clumped into pollinia (Johnson and Edwards 2000). In most rewardless orchids pollination does not rely on mimicry (Ackerman 1986; Nilsson 1992) but appears to be effected by naïve pollinators exploring their environment and sampling rewardless inflorescences before switching to more profitable food sources (Nilsson 1992).

In entomophilous rewardless species the effects of spatial arrangement on pollinator behaviour, e.g. the level of aggregation, within a plant community should differ from those of rewarding plants. Pollinators are expected to change their flight distances and visitation rates in response to both the availability and the quantity of rewards (Stephen and Krebs 1986). They are likely to leave quickly areas with few flowers, either rewarding or rewardless, and remain for longer in patches that provide a high reward or where rewarding flowers are highly aggregated (Real 1983). A high level of aggregation of rewardless plants may reduce pollinator visitation, because pollinators learn to avoid them more quickly, whereas a high level of aggregation of rewarding co-flowering plants may increase it. Other types of pollinator behaviour can change in response to amount of reward: after encountering a rewardless plant, a pollinator increases both its flight distance to the next inflorescence (Dukas and Real 1993) and the probabilities of sampling a flower of a different phenotype on the next visit (Smithson and Macnair 1997). The pollinator continues this process until it encounters a rewarding flower, after which the probability of visiting other individuals of the same phenotype increases (Smithson and Macnair 1997; Cartar 2004). Previous studies suggest that pollinators may show short-term avoidance of unrewarding morphs and longterm memorising of rewarding morphs (Dukas and Real 1993; Smithson and Macnair 1997). The spatial aggregation of rewarding and rewardless plants influences the probabilities of encountering and visiting both plant types, which in turn affect pollinator learning. As a consequence, the spatial aggregation of rewarding and rewardless flowers should influence the reproductive success of rewardless plants, so that components of reproductive success in rewardless species should change with variation in their levels of aggregation.

A large body of theory indicates the importance of spatial patterns in ecology (Tilman and Kareiva 1997; Dieckmann et al. 2000). Although empirical studies on competition in plants are abundant, very little is known about how the spatial structure of plant communities influences inter- and intra-specific interactions (Goldberg and Barton 1992; Gurevitch et al. 1992). Facilitation among plants for pollinator attraction has been shown (Waser and Real 1979; Thomson 1981, 1982) and discussed (Rathcke 1983; Real 1983), as well as the influence of plant density on pollinator visitation rate (Schmitt 1983a, b; Waddington 1980; Johnson et al. 2003; Alexandersson and Àgren 1996; Gumbert and Kunze 2001). These studies suggest that, in the presence of competitor plants, pollinator visitation can increase in some cases and decrease in others. Similarly, increased density of co-flowering species can increase or decrease visitation rate. Finally, increased density of a focal species can diminish, increase or have no effect on pollinator visitation rate. However, the effects of spatial aggregation of plant species on pollinator visitation within a plant community have not, to our knowledge, been experimentally documented. In an experimental context the concepts of density and spatial aggregation are particularly difficult to separate, because variation in density may be correlated with variation in spatial aggregation.

Very little is known about both the effect of rewardless plant spatial aggregation and that of coflowering species on the reproductive success of the rewardless plants. To our knowledge, there is only one experimental study, based on an artificial single flower system, investigating the effect of spatial distribution of rewardless and rewarding species on visitation rate (Keasar 2000). This study showed that the proportion of moves from rewarding to unrewarding flowers, and the total number of visits to unrewarding flowers, were significantly higher when distributed in monospecific patches than in a randomly intermingled distribution. However, the effects of intermingling and spatial aggregation were potentially confounded, and, further, results from such artificial systems do not necessarily reflect patterns obtained from natural populations.

In the present study we experimentally addressed whether the reproductive success of a rewardless orchid may be affected by its own aggregation and that of a rewarding co-flowering species, using a full factorial experimental design combining two relative levels of aggregation. We hypothesised, on the basis 
of expected pollinator behaviour patterns, that an increase in aggregation should decrease the reproductive success of the rewardless species, because pollinators will avoid such a patch when encountered. For the same reasons, we also predicted that increasing aggregation of rewarding plants would increase reproductive success of the intermingled rewardless plants, because a dense rewarding patch will attract more pollinators and, consequently, might also increase the reproductive success of surrounding rewardless plants.

\section{Material and methods}

Study system

We used the widespread rewardless orchid Dactylorhiza sambucina Soó as a model study system. $D$. sambucina has two co-occurring corolla colour morphs, purple and yellow, but only the yellow morph was used in this study to avoid possible confounding effects of colour polymorphism with aggregation. Further, the yellow morph of the species is much more common in the study area than the purple one. D. sambucina flowers in April and May and is pollinated by newly emerged bumblebee queens (Nilsson 1980). Its flowers are zygomorphic, with an 8-12 mm long labellum, and grouped into a dense inflorescence. We selected Muscari neglectum Tenore (Asparagaceae) as our experimental nectar-rewarding species, because it naturally co-flowers with $D$. sambucina and is visited by the same pollinators (L.D.B. Gigord and A. Smithson, personal observation). Pollinators are likely to distinguish the two species easily: flowers of $M$. neglectum are very small, actinomorphic, grouped into a dense inflorescence and are deep blue in colour, thus mimicry could be excluded as a potential confounding factor.

\section{Study site}

We carried out the experiment in southern France

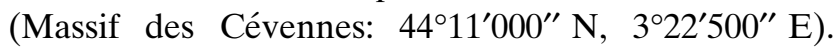
Two hundred and forty yellow D. sambucina plants were carefully excavated, temporarily potted, and bagged at bud stage. Each orchid plant and its original position were marked in order to replace it at the end of the experiment. Seven hundred and twenty $M$. neglectum plants were also excavated and temporarily potted. The other few co-flowering species, apart from $D$. sambucina and $M$. neglectum, surrounding the experimental arrays were mainly pollen rewarding.
Experimental design

We used a full factorial experimental design with two levels of aggregation for the two species, with four replicates per treatment combination, giving a total of 16 arrays (Fig. 1). These arrays were set up in a homogeneous habitat, similar to the natural habitat of these species (open or semi-open meadows). Naturally occurring $M$. neglectum inflorescences within and surrounding the array-up to $1 \mathrm{~m}$ distance from the edge-were removed. Each array contained 30 potted plants: 15 pots with one $D$. sambucina per pot and 15 pots with three $M$. neglectum per pot, to give a similar number of open flowers for the two species (see Fig. 1 for details). The same numbers of individuals of the two species were used in each array to avoid frequency-dependent patterns of pollinator visitation. Aggregation treatments and pots were randomly allocated to arrays and within each of them, respectively. The orchid plants used in the experiment had 7-14 flowers $(\bar{x}=9.23 \pm \mathrm{SE}=0.12)$, and were allocated to arrays to ensure that similar numbers of flowers were available per array $(\bar{x}=138.5 \pm \mathrm{SE}=0.13)$. The number of flowers per plant did not differ significantly among arrays, either at the beginning (Kruskal-Wallis chi-squared $=0.03, d f=15, P=1, n=16)$ or at the end (Kruskal-Wallis chi-squared $=10.58, d f=15, P=0.78$,

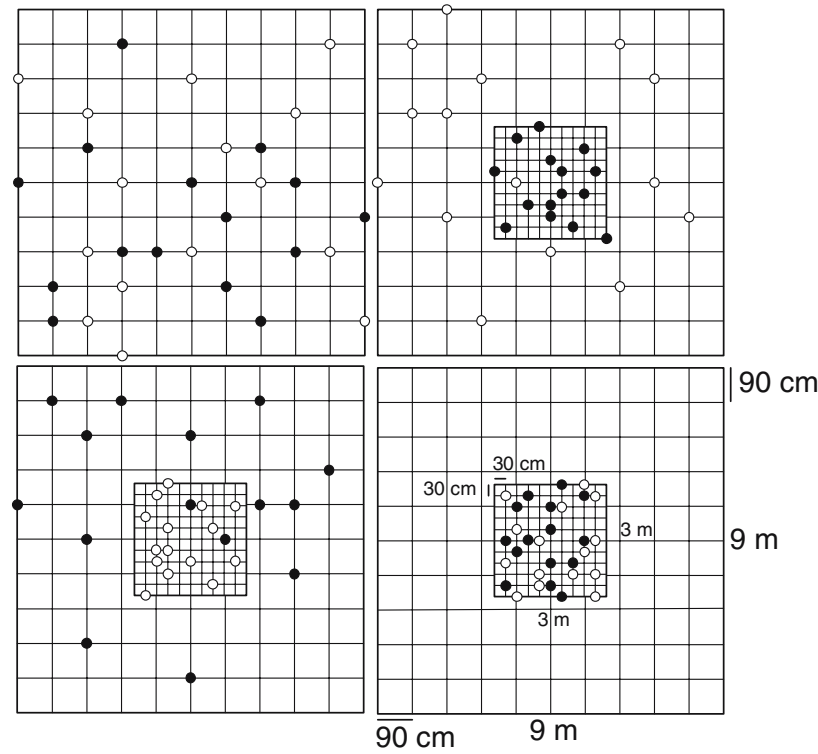

Fig. 1 Scheme of the experimental design showing the four aggregation treatments. For low aggregation levels of Dactylorhiza sambucina (open circles) and Muscari neglectum (filled circles), pots were distributed in a $9 \mathrm{~m} \times 9 \mathrm{~m}$ array. For high aggregation levels, pots were allocated in a $3 \mathrm{~m} \times 3 \mathrm{~m}$ area located in the centre of the large one. The grids represent 121 potential positions for high and low aggregation levels 
$n=16$ ) of the experiment. To ensure that experimental conditions were maintained through the experiment, we replaced damaged or wilted plants $\bar{x}=3.88 \pm \mathrm{SE}=$ 0.65 per array) with plants with corresponding numbers of flowers. Orchids that were replaced were not included in reproductive success estimation and analysis. Distance between two neighbouring plants in our experimental arrays ranged between $30 \mathrm{~cm}$ and $460 \mathrm{~cm}$. In natural populations these values vary between $2 \mathrm{~cm}$ and $1,510 \mathrm{~cm}$ (L.D.B. Gigord and A. Smithson, unpublished data). Thus, levels of aggregation in the experiment were biologically relevant.

\section{Data collection}

We exposed plants simultaneously to pollinators on the evening of 14 May 2004, when all orchids had at least five open flowers. Plants were watered daily during the period of exposure. After 8 days of exposure, all $D$. sambucina plants were bagged, and male reproductive success (pollinia removal, i.e. the number of pollinia removed divided by the total number of pollinia available for removal) was estimated for each individual. After 5 weeks, fruit set was counted for $D$. sambucina and female reproductive success determined as the number of fruits produced divided by the total number of flowers available. Reproductive success was then averaged in each array.

\section{Data analysis}

Differences in male and female reproductive success among treatments and arrays were analysed by a mixed analysis of variance (ANOVA) model with permutation tests on the mean squares (Manly 1997), since residuals violated ANOVA assumptions of normality and homoscedasticity. The two response variables were pollinia removal and fruit set, whereas the explanatory variables were aggregation level of $D$. sambucina, aggregation level of $M$. neglectum, and array nested in the interaction between the two aggregation variables.

We estimated effects of aggregation by permuting aggregation levels in the data set separately for each of the two species. The interaction between the levels of aggregation of the two species was tested by permutation of aggregation levels in the data set for both species at the same time. Finally, we tested the replication level (i.e. array) by permuting array numbers within the combination of the levels of aggregation of the two species. $P$ values were computed for each treatment as the proportion of permuted mean-square estimates larger than or equal to observed mean square over 10,000 permutations. All statistical analyses were conducted with R 1.9.1 software (R Development Core Team 2004). Throughout the results, mean values are given with their standard errors.

\section{Results}

Increased aggregation of $D$. sambucina marginally reduced pollinia removal and significantly reduced fruit set (Table 1, Fig. 2). Increased aggregation of $M$. neglectum marginally reduced pollinia removal of $D$. sambucina and significantly reduced its fruit set (Table 1, Fig. 2). Thus, high levels of aggregation of both the rewardless and the rewarding species significantly reduced $D$. sambucina reproductive success, in particular through female function.

The interaction between the levels of aggregation of the two species had no significant effect on pollinia removal and a marginally significant effect on fruit set (Table 1, Fig. 2). Array had no significant effect on male reproductive success but had a significant effect on female reproductive success (Table 1).

\section{Discussion}

In a controlled manipulative field experiment, fruit set of $D$. sambucina decreased as its spatial aggregation increased, regardless of the aggregation level of $M$. neglectum, which supported our a priori prediction. We also found that the lower the aggregation of $M$. neglectum, the higher the fruit set of $D$. sambucina, which

Table 1 Effects of Dactylorhiza sambucina and Muscari neglectum aggregation levels, of their interaction $(D$. sambucina $\times M$. neglectum) and of array [array in (D. sambucina $\times M$. neglectum)] on (a) male (proportion of pollinia removed) and (b) female (proportion of fruit produced) reproductive success of D. sambucina, tested with a mixed ANOVA with permutation method

\begin{tabular}{lrll}
\hline Source of variation & $d f$ & MS & \multicolumn{1}{l}{$P$} \\
\hline (a) Male reproductive success & & & \\
D. sambucina & 1 & 0.1894 & $0.0516^{\dagger}$ \\
M. neglectum & 1 & 0.1691 & $0.0609^{\dagger}$ \\
D. sambucina $\times$ M. neglectum & 1 & 0.0246 & 0.4896 \\
Array in & 12 & 0.0911 & 0.1125 \\
$\quad$ D. sambucina $\times$ M. neglectum) & & & \\
(b) Female reproductive success & & & \\
D. sambucina & 1 & 0.6173 & $<0.0001^{* * *}$ \\
M. neglectum & 1 & 0.2196 & $0.0082^{* *}$ \\
D. sambucina $\times$ M. neglectum & 1 & 0.1069 & $0.0835^{\dagger}$ \\
Array in & 12 & 0.0997 & $0.0123^{*}$ \\
$\quad(D$. sambucina $\times$ M. neglectum $)$ & & & \\
\hline
\end{tabular}

${ }^{\dagger} P<0.1, * P<0.05, * * P<0.01, * * * P<0.001$ 


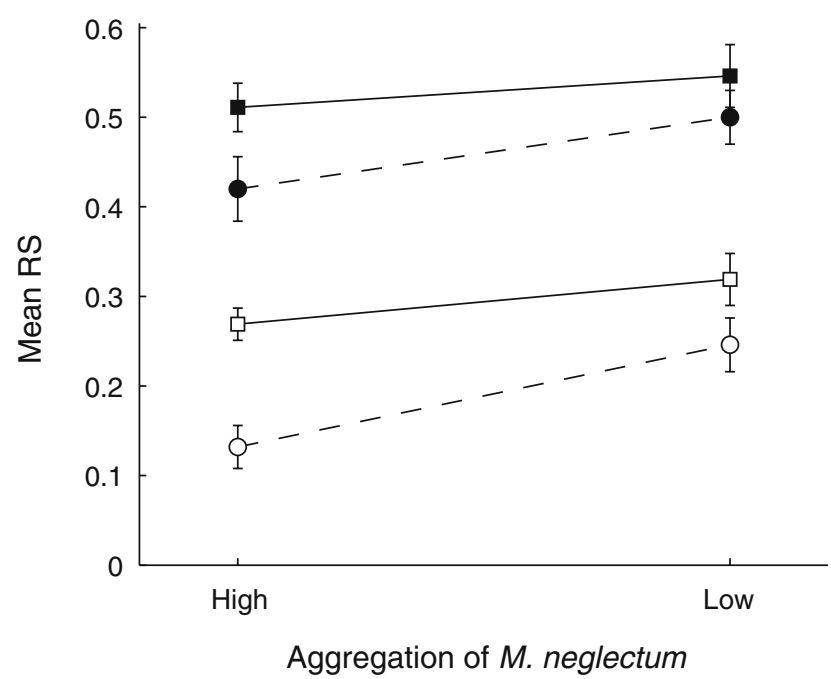

Fig. 2 Mean male (filled symbols) and female (open symbols) reproductive success of $D$. sambucina according to aggregation levels of $M$. neglectum and of D. sambucina (squares and circles represent low and high aggregation, respectively). Bars represent standard errors

did not support our a priori prediction. We expected that specific pollinator behaviours would affect avoidance learning of rewardless plants through different mechanisms, for example, by reducing time spent by pollinators in a patch offering a lower amount of reward (Real 1983; Dukas and Real 1993), increasing flight distances and visitation rates after a rewardless visit (Real 1983; Stephen and Krebs 1986; Smithson and Macnair 1997), or through short-term avoidance of rewardless species and long-term memorising of rewarding species (Dukas and Real 1993; Smithson and Macnair 1997). Each of these behaviours is expected, in turn, to depend on the relative encounter and, consequently, on the relative visitation rates to the rewarding and rewardless plants. As these rates depend on the relative spatial aggregation of both types of species, the differences in reproductive success found in this study should be a direct consequence of the spatial aggregation of rewarding and rewardless plants. As predicted, a high level of spatial aggregation of the orchid may increase the probability that pollinators quickly leave the patch, thereby lowering the reproductive success of the rewardless species. The higher the level of aggregation of rewardless plants, the more often pollinators encounter them and the faster they may learn to avoid them. This is supported by Gumbert and Kunze (2001), who showed a significant negative effect of density of the rewardless orchid Orchis boryi on its fruit production in $1 \mathrm{~m}^{2}$ plots.

How can we explain the result that the reproductive success of $D$. sambucina increased with decreased aggregation of $M$. neglectum? High levels of aggregation in rewarding species might encourage pollinators to stay in the patch for longer, but may simultaneously increase interspecific competition for access to pollinators, thus decreasing visitation to the rewardless species. Pollinators may learn and favour rewarding species in the long term (Dukas and Real 1993) and, consequently, visit $M$. neglectum flowers preferentially, since the rewardless species will be the poorer competitor for pollinator visitations. This could explain the decrease of $D$. sambucina reproductive success with the increased aggregation of M. neglectum.

We deliberately chose easily distinguishable rewarding and rewardless species. This dissimilarity, which facilitates the discrimination of the two species by pollinators, might also reinforce avoidance learning and reduce the likelihood that a pollinator would move from a rewarding to a rewardless plant, especially at high aggregation of $M$. neglectum. Bumblebees preferentially move to flowers of similar colour to those on which they have recently been foraging with success (Smithson and Macnair 1996; Gumbert and Kunze 2001; Gigord et al. 2002). Because D. sambucina and $M$. neglectum are clearly dissimilar in several floral traits, including corolla colour, the higher the aggregation of $M$. neglectum, the higher might be the probability that pollinators visit mainly $M$. neglectum plants. In this situation pollinators can focus on the rewarding species while maintaining a low cost of flight. When not rewarded, pollinators are more likely to move to flowers of a different colour and, consequently, avoid flowers of the rewardless species (Smithson and Macnair 1997). Thus, rewardless species are probably more likely to benefit from flowering at the same time as rewarding species of similar corolla colour (Gumbert and Kunze 2001), while negative effects such as those we observed may principally occur for dissimilar corolla colour species pairs. Supporting this hypothesis, Johnson et al. (2003) found that increased density of the rewarding Allium schoenoprasum (similar corolla colour) strongly enhanced the reproductive success of the rewardless Anacamptis morio, but that increased density of the rewarding Lotus corniculatus (different corolla colour) slightly decreased it. This is in accordance with the decrease of fruit set with increased aggregation of the dissimilar $M$. neglectum found here. However, high aggregation of a rewarding co-flowering species cannot explain why, in some studies, the reproductive success of rewardless orchids increased with increasing rewarding species aggregation, even when corolla colours were dissimilar (Alexandersson and ̊gren 1996; Gumbert and Kunze 2001). Our results also contrast with those of Keasar 
(2000), who showed that the total number of visits to unrewarding types was significantly higher in a patchy flower arrangement, where rewarding and rewardless plants were aggregated but spatially separated, than in an intermingled one, where plants were dispersed but mixed. We, however, obtained the highest reproductive success for a low level of aggregation of the two intermingled species and the lowest reproductive success when $M$. neglectum and $D$. sambucina were intermingled at a high level of aggregation. As the rewarding and rewardless plants were either intermingled or separated in Keasar's study, the effect of aggregation was possibly confounded with an intermingling effect, which is not the case in the present study. This suggests that the effects of aggregation of rewarding and rewardless species remain unclear and could depend both on the species pair that is considered and the local spatial arrangement of plant species (intermingled or separated). More experiments are necessary to distinguish the effects of intermingling and aggregation.

This experiment shows that high levels of aggregation of both a rewardless and a rewarding species that are phenotypically dissimilar diminishes the fruit set of the rewardless species, mediated by changes in pollinator visitation. These results imply that it is fundamental to take into account the spatial aggregation of both co-flowering species and the focal rewardless species when assessing their reproductive success. These results could also explain why rewardless orchids flower early in the season, which may be the result of selection for increased pollinator services. The detrimental effects of high aggregations of both species should be considered when reintroducing rewardless orchids in a natural environment. However, as other factors such as colour similarity between the rewarding and the rewardless species and their intermingling seem to interact with the aggregation effects, more experiments are needed to understand the effects of aggregation sensu lato. Because of the complexity of aggregation effects and interactions among plant species and pollinators, similar in situ large-scale experimental approaches are fundamental for obtaining a better understanding of these natural phenomena.

Acknowledgements This research was supported by the Swiss National Science Foundation research grant reference 3100A0$100754 / 1$ to Luc D. B. Gigord, and the Roche Research Foundation Grant 22-2004 to Giorgina Bernasconi and Luc D. B. Gigord. Ann Smithson was funded by a British Ecological Society Special Ecological Project grant on the conservation of rewardless orchids. We are very grateful to Mailyn Gonzalez, Aline Pasche, Tiziana Ulian, Yves Delacrétaz, Javier Vences, Christine Gruffaz and the Baraille and Passet families for their inestimable support with the experiment. We also thank
Giorgina Bernasconi, Guillaume Evanno and Pascal Vittoz for constructive comments on early versions of the manuscript.

\section{References}

Ackerman JD (1986) Mechanisms and evolution of food deceptive pollination systems in orchids. Lindleyana 1:108-113

Alexandersson R, Agren J (1996) Population size, pollinator visitation and fruit production in the deceptive orchid $\mathrm{Ca}$ lypso bulbosa. Oecologia 107:533-540

Callaway RM (1995) Positive interactions among plants. Bot Rev 61:306-349

Cartar RV (2004) Resource-tracking by bumble bees: responses to plant-level differences in quality. Ecology 85:2764-2771

Dieckmann U, Law R, Metz JAJ (2000) The geometry of ecological interactions: simplifying spatial complexity. Cambridge University Press, Cambridge

Dukas R, Real LA (1993) Effects of recent experience on foraging decisions by bumblebees. Oecologia 94:244-246

Gigord LDB, Macnair MR, Stritesky M, Smithson A (2002) The potential for floral mimicry in rewardless orchids: an experimental study. Proc R Soc Lond Ser B 269:1389-1395

Goldberg DE, Barton AM (1992) Patterns and consequences of interspecific competition in natural communities: a review of field experiments with plants. Am Nat 139:771-801

Gumbert A, Kunze J (2001) Color similarity to rewarding model plants affects pollination in a food deceptive orchid, Orchis boryi. Biol J Linn Soc 72:419-433

Gurevitch J, Morrow L, Wallace A, Walsh JS (1992) A metaanalysis of competition in field experiments. Am Nat 140:539-572

Holzapfel C, Mahall BE (1999) Bidirectional facilitation and interference between shrubs and annuals in the Mojave desert. Ecology 80:1747-1761

Johnson SD, Edwards T (2000) The structure and function of orchid pollinia. Plant Syst Evol 222:243-269

Johnson SD, Peter CI, Nilsson LA, Ågren J (2003) Pollination success in a deceptive orchid is enhanced by co-occurring rewarding magnet plants. Ecology 84:2919-2927

Keasar T (2000) The spatial distribution of nonrewarding artificial flowers affects pollinator attraction. Anim Behav 60:639-646

Kennedy M, Gray RD (1993) Can ecological theory predicts the distribution of foraging animals? A critical analysis of experiments on the Ideal Free Distribution. Oikos 68:158166

Kunin WE (1993) Sex and the single mustard: population density and pollinator behavior effects on seed-set. Ecology $74: 2145-2160$

Manly BFJ (1997) Randomization, bootstrap and Monte Carlo methods in biology. Chapman and Hall, London

Moeller DA (2004) Facilitative interactions among plants via shared pollinators. Ecology 85:3289-3301

Nilsson LA (1980) The pollination ecology of Dactylorhiza sambucina (Orchidaceae). Bot Notiser 133:367-385

Nilsson LA (1992) Orchid pollination biology. Trends Ecol Evol 7:255-259

Pacala SW (1997) Dynamics of plant communities. In: Crawley MJ (ed) Plant ecology. Blackwell Scientific, Oxford, pp 532555

R Development Core Team (2004) R: A language and environment for statistical computing. R Foundation for Statistical Computing, Vienna, Austria. ISBN 3-900051-00-3. URL: http://www.R-project.org 
Rathcke B (1983) Competition and facilitation among plants for pollination. In: Real L (ed) Pollination biology. Academic, New York, pp 309-329

Real L (1983) Microbehavior and macrostructure in pollinator plant interactions. In: Real L (ed) Pollination ecology. Academic, New York, pp 287-302

Schmitt J (1983a) Flowering plant density and pollinator visitation in Senecio (Compositae). Oecologia 60:97-102

Schmitt J (1983b) Density-dependent pollinator foraging, flowering phenology, and temporal pollen dispersal patterns in Linanthus bicolor. Evolution 37:1247-1257

Smithson A, Macnair MR (1996) Frequency-dependent selection by pollinators: mechanisms and consequences with regard to behavior of bumblebees Bombus terrestris (L.) (Hymenoptera: Apidae). J Evol Biol 9:571-588

Smithson A, Macnair MR (1997) Negative frequency-dependent selection by pollinators on artificial flowers without rewards. Evolution 51:715-723

Stephen DW, Krebs JR (1986) Foraging theory. Princeton University Press, Princeton

Thomson JD (1978) Effect of stand composition on insect visitation in two-species mixtures of Hieracium. Am Midl Nat 100:431-440
Thomson JD (1981) Spatial and temporal components of resource assessment of flower-feeding insects. J Anim Ecol 50:49-59

Thomson JD (1982) Patterns of visitation by animal pollinators. Oikos 39:241-250

Thomson JD (1983) Component analysis of community-level interactions in pollination systems. In: Jones CE, Little RJ (eds) Handbook of experimental pollination biology. Van Nostrand Reinhold, New York, pp 451-460

Tilman D, Kareiva P (1997) Spatial ecology: the role of space in population dynamics and interspecific interactions. Princeton University Press, Princeton

Tirado R, Pugnaire FI (2003) Shrub spatial aggregation and consequences for reproductive success. Oecologia 136:296301

van der Pijl L, Dodson CH (1966) Orchid flowers, their pollination and evolution. University of Miami Press, Coral Gables

Waddington KD (1980) Flight patterns of foraging bees relative to density of artificial flowers and distribution of nectar. Oecologia 44:199-204

Waser NM, Real L (1979) Effective mutualism between sequentially flowering plant species. Nature 281:670-672 